\title{
Network Structure Oriented Evolutionary Model:
}

\section{Genetic Network Programming \\ -Its Comparison with Genetic Programming-}

\author{
Hironobu Katagiri*, Kotaro Hirasawa*, Jinglu Hu*, \\ Junichi Murata* and Michitaka KosaKA**
}

\begin{abstract}
Recently many studies have been made on automatic design of complex systems by using evolutionary optimization techniques such as Genetic Algorithm (GA), Genetic Programming (GP) and Evolutionary Programming (EP). In this paper, a new method named Genetic Network Programming (GNP) is proposed in order to develop a more effective evolutionary optimization technique. GNP is composed of plural nodes which execute simple judgement/processing and they are connected with each other. To put it another way, GNP forms network structures, while general GPs form tree structures. This paper shows the detailed description about GNP and points out the differences among GNP, GP and other conventional graph based methods. The comparison between GNP and GP is also shown by using a virtual simulation, the tileworld.
\end{abstract}

Key Words: evolutionary computation, network structure, genetic programming, planning, tileworld

\section{Introduction}

A large number of studies have been made on the evolutionary optimization techniques such as Genetic Algorithm $(\mathrm{GA})^{1)}$, Genetic Programming $(\mathrm{GP})^{2), 3)}$ and Evolutionary Programming $(\mathrm{EP})^{4), 5)}$. GA evolves strings and can find suboptimal solutions of the problems quickly, so it has been widely studied and applied to many real optimization problems. GP was devised later in order to expand GA's representation ability by using tree structures as substitution for GA's strings. This structural change of solutions brought about more progress to evolutionary computation and made GP applicable to more complex problems. Therefore, it is important how to represent the solution for the optimization problems, because the form of the solutions restricts the problems to be applicable.

In this paper, a new method named Genetic Network Programming (GNP) is proposed. GNP is also one of the evolutionary optimization techniques, which uses network structure as solutions. GNP is composed of many and various nodes. These nodes are roughly classified into two kinds of nodes: JUDGEMENT NODE and PROCESSING NODE. JUDGEMENT NODEs correspond to some concrete functional

* Graduate School of Information Science and Electrical Engineering, Kyushu University, 6-10-1, Hakozaki, Higashi-ku, Fukuoka

** Hitachi Ltd. System Development Laboratory, 1099 Ozenji, Asao-ku, Kawasaki

(Received June 28, 2001)

(Revised March 15, 2002) nodes which work as judgements. They correspond nearly to GP's elementary functions. PROCESSING NODEs work as actions. They correspond almost to GP's terminal symbols. The individuals are represented by connecting these nodes with each other.

There have been studied many network structure oriented (graph-based) evolutionary methods. However, GNP clearly differs from these methods. We describe the differences between GNP and conventional methods in section 4. 3. This paper tries to develop an evolutionary method with network structure and study the differences between the network structure and the tree structure. Although each method has its favorite problems and the problems hard to apply, the simulation results show one of the advantages of the network structure over the tree structure in a specific simulation environment. The simulation environment is the tileworld which is well known as the test bed problem.

This paper is organized as follows. In the next section, conventional graph based evolutionary methods are introduced briefly. Section 3 describes the problem used in the simulations. Section 4 describes the detail of Genetic Network Programming, Section 5 shows the comparison of GNP with GP, and finally some conclusions are given in Section 6.

\section{Conventional graph based evolution- ary methods}

In this section, the conventional graph based evolution- 
ary methods are briefly introduced. The different points from the proposed method are argued in section 4 after the explanation of GNP.

\section{1 Parallel Algorithm Discovery and Orches-} tration ${ }^{6)}$

Parallel Algorithm Discovery and Orchestration (PADO) is one of the graph based GP instead of the tree structure. This is specific in the point of using stack memory and indexed memory because it is an approach based on the mental model. PADO has three main components, a main program, ADF programs, and indexed memory. In the main program, there are a start node and an end node. ADF is well known in GP, which is the function set defined automatically in the program runs. The execution of PADO is carried out from the start node to the end node in the network. PADO was applied to the object recognition problems.

\section{2 Cartesian Genetic Programming ${ }^{7)}$}

Cartesian Genetic Programming (CGP) tried to explore the graph based GP motivated by the general representation ability of the graph compared with the tree. Its program is represented as an indexed graph. CGP adopts the genotype-phenotype mapping. The genotype is an integer string which denotes a list of node connections and functions. This string is mapped into phenotype of an indexed graph. It is insisted in the paper on CGP that the redundancy enhances the evolutionary ability.

\section{3 Evolutionary Programming ${ }^{4), 5)}$}

Evolutionary Programming (EP) is also the networkstructured system developed by L. J. Fogel. EP is one of the evolutionary computations like GA and GP, so its concept is almost the same as GA and GP, but generally EP uses only mutation as evolutionary operators. It is used for the automatic synthesis of Finite State Machines, which was originally used to solve sequence prediction problems.

\section{The Problem}

We describe the simulation environment in advance to explain the following section easily.

\section{1 The Tileworld}

The tileworld ${ }^{8) \sim 10)}$ is a virtual environment for agents in 2-dimentional latticed world, which is well known as a testbed for studying agents' behaviors in dynamical environments. The tileworld consists of some agents, tiles, barriers, holes and floors. Fig. 1 shows ten kinds of tileworlds used in the simulations. The left top environment in Fig. 1 is borrowed from 9), and others are created by changing the location of tiles and holes slightly. There are
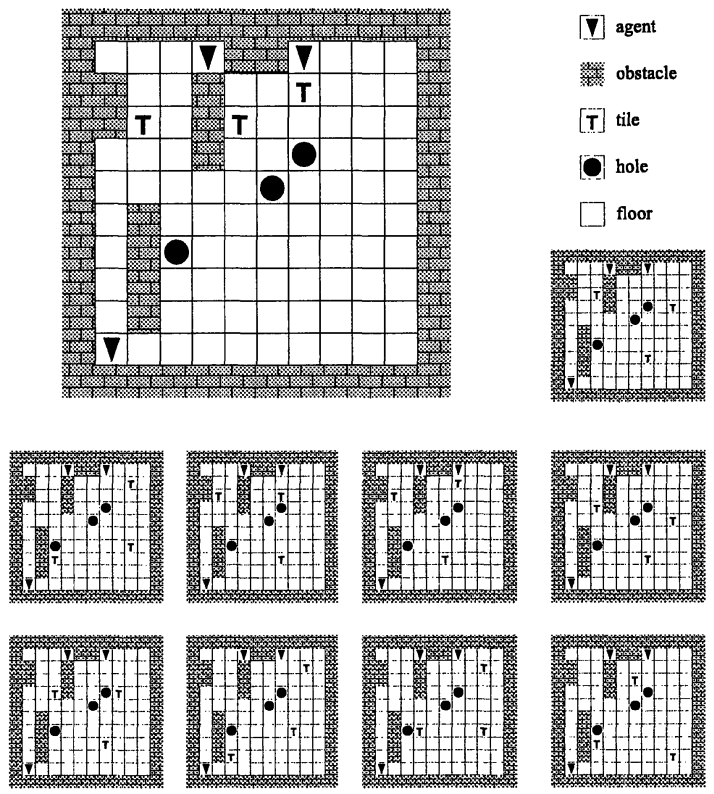

Fig. 1 The tileworlds

three agents, three tiles and three holes in the environment, respectively. The tileworld is a Partially Observable Markov Decision Process (POMDP) problem, provided the agents cannot utilize the whole information of the world.

In this world, agents can move to the neighboring floor square in one step period. Moreover, the agent can push the tile to the floor square except where there are the barriers or other agents exist. When the tile is pushed into the hole, the hole and the tile will vanish, namely, the hole is plugged with the tile. The purpose of the agents is to drop all tiles into holes as early as they can.

Here, agents can only move to front square (MF), turn right (TR), turn left (TL), and stay (ST). In other words, agents have the sense of direction to move.

Agents can find out what exists at the square in front of each agent (CF), in the same way, right (CR), left (CL), and back (CB) of each agent. Agents can also find out the rough direction from the agents to the place where the nearest tile is (NTD), where the second nearest tile is (SNT), and where the nearest hole is (NHD). Furthermore, they can find out the rough direction from the nearest tile of the agent to the square where the nearest hole is (HTD). The rough direction here is defined as the following four directions: front, right, left and back. Agents are not informed of other information, e.g., the coordinate information, the distance information, and the angle information. This is very difficult problem as if men find their way with a few sensors in a dark environment. Agents should ac- 


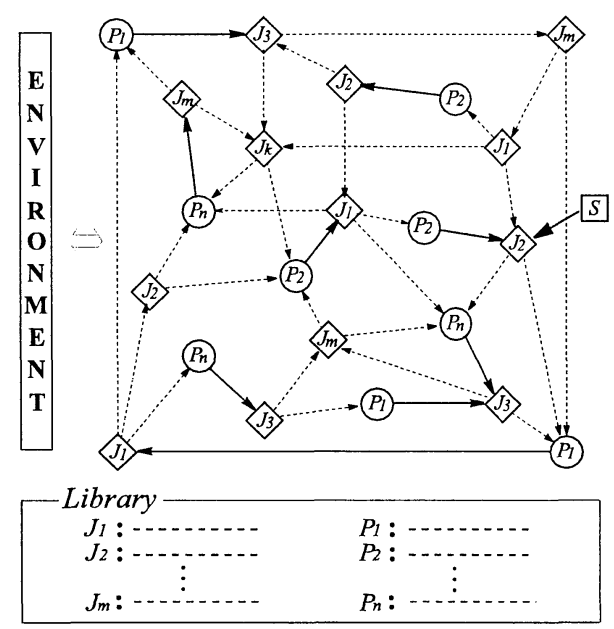

Fig. 2 The basic structure of GNP individual

quire the following knowledge: what judgement should be done, and what to do according to the judgement result.

\section{Genetic Network Programming ${ }^{11) ~ 14)}$}

In this section, Genetic Network Programming (GNP) is explained in detail. Basically, GNP is an extension of GP in terms of gene structures. The original idea is based on the more general representation ability of graphs than that of trees.

\subsection{Basic structure of GNP}

The basic structure of GNP is shown in Fig. 2. As shown in Fig. 2, the network structure is used to represent individuals. GNP is composed of plural nodes which are connected with each other like networks. These nodes are roughly classified into two kinds of nodes: JUDGEMENT NODE and PROCESSING NODE.

JUDGEMENT NODEs correspond nearly to elementary functions of GP and PROCESSING NODEs correspond almost to terminal symbols of GP. JUDGEMENT NODEs are the set of $J_{1}, J_{2}, \cdots, J_{m}$, which work as some kinds of judging functions. On the other hand, PROCESSING NODEs are denoted by the set of $P_{1}, P_{2}, \cdots, P_{n}$, which work as some kinds of action/processing functions. The practical roles of these nodes are predefined and stored in the library by supervisors.

GP's elementary functions and terminal symbols are repeatedly used in a tree structure. In the same way, there are some $J_{1} \mathrm{~s}, J_{2} \mathrm{~s}, P_{1} \mathrm{~s}, P_{2} \mathrm{~s}$ and so on in GNP as shown in Fig. 2. These JUDGEMENT NODEs and PROCESSING NODEs are the essential elements of GNP. The number of these nodes may be determined as a result of evolution like GP. Actually, GNP can use this strategy, in other words, GNP

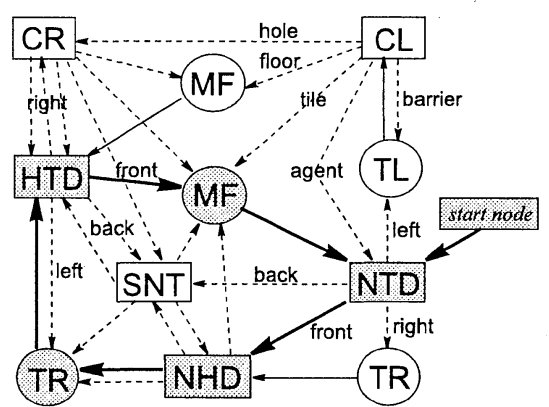

Fig. 3 A manually coded GNP network

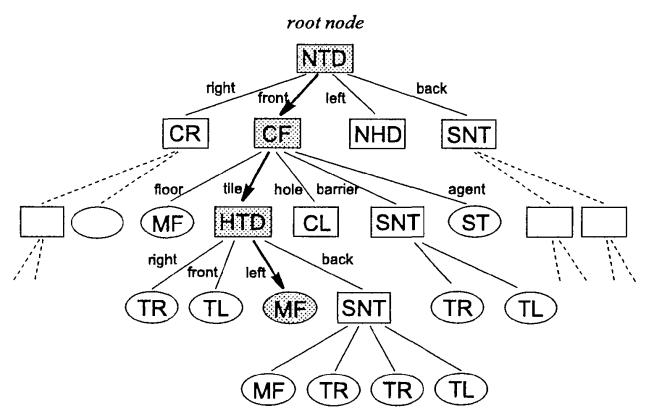

Fig. 4 A manually coded GP tree

can adopt evolving the genotypes with variable number of nodes, but in this paper GNP evolves only the networks with the predefined number of nodes. It would be better to say that GNP here evolves the genotypes with fixed number of nodes. We set the number of each node in GNP equal to each other, e.g., $J_{1} \times 5, J_{2} \times 5, \cdots, P_{1} \times 5$, $P_{2} \times 5, \cdots$, and so on.

Additional one specific node, start node $S$, is involved in GNP. Start node indicates the start point of GNP, which corresponds to GP's root node.

Conventional tree based GPs start from its root node, then evaluate leaf nodes one after another. Although there are many GP studies using the numerical information and studies using multiple-choice information, GNP uses the multiple-choice information in JUDGEMENT NODE, does not use numerical information. Namely, JUDGEMENT NODEs here are if-then type decision making functions.

Once GNP is booted up, the execution starts from start node, then the next node to be executed is determined according to the connection from the current activated node. If the activated node is JUDGEMENT NODE, the next node is determined by the judgement at the activated JUDGEMENT NODE. When PROCESSING NODE is executed, the next node is uniquely determined by the single connection from PROCESSING NODEs.

Fig. 3 shows a manually coded GNP network under the tileworld problem. Nodes surrounded by boxes are 


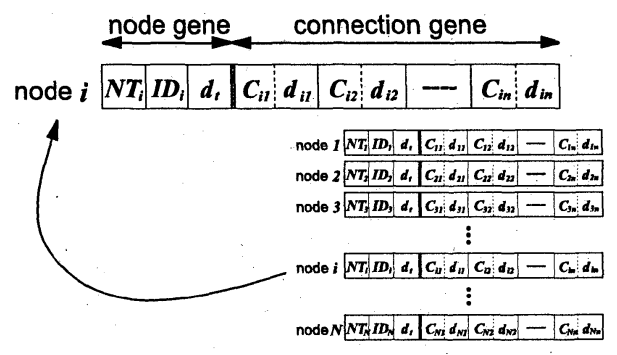

Fig. 5 The genotype expression of GNP node

JUDGEMENT NODEs and nodes surrounded by circles are PROCESSING NODE. An example of the decision making process is emphasized by shadowed boxes, circles and bold lines. Here; the small letters nearby the arrows are the labels corresponding to the judgement. In the beginning, the start node is activated. Next, NTD is activated, where an agent judges the rough direction (right, front, left, and back) to the nearest tile. The next node is determined according to the judgement result. In Fig. 3 , the judgement result is "front", then NHD is activated. In the same way, TR is activated according to the judgement result at NHD. TR is a PROCESSING NODE, then agent turns to the right. The next activated node is uniquely determined to be HTD. In this way, the activated node is transferred one after another.

For comparison, an example of the manually coded GP tree in the simulation are shown in Fig. 4. It is interpreted from the root node. The emphasized boxes, circles and arrows in Fig. 4 show the decision making process of an agent as an example. The tree is interpreted from the root node over and over again every steps of the processing of agents.

It is an important point of GNP that the activated node isn't compulsorily transferred to the start node, while other methods do. For instance, GP reinterpret the tree again from the root node after completing the interpretation of the tree, and PADO individuals have both a start node and an end node. There are no end node in GNP, therefore, once GNP is booted up, the successive activation of GNP system is carried out according to the network flow.

The genotype expression of GNP node is shown in Fig. 5. This describes the gene of node $i$, then the set of these genes represents the genotype of GNP individuals. All variables in these genes are described by integer. $N T_{i}$ describes the node type, $N T_{i}=0$ when the node $i$ is JUDGEMENT NODE, $N T_{i}=1$ when the node $i$ is PROCESSING NODE. $I D_{i}$ is an identification number, e.g., $N T_{i}=0$ and $I D_{i}=1$ mean node $i$ is $J_{1} . C_{i 1}, C_{i 2}, \cdots$, denote the nodes which are connected from node $i$ firstly, secondly, $\cdots$, and so on depending on the arguments of node $i$. The total number of connection genes depends on the arity of the node's function. $d_{i}$ and $d_{i j}$ are the delay time. They are the time required to execute the processing of node $i$ and delay time from node $i$ to node $C_{i j}$, respectively. GNP can become materialized more realistically by setting these delays.

\subsection{Genetic operator of GNP}

The following genetic operators shown in Fig. 6, 7 are used in GNP. Mutation operator affects one individual. All the connections of each node are changed randomly by mutation rate of $P_{m}$ (shadowed in Fig. 6). Crossover operator affects two parent individuals. All the connections of the uniformly selected corresponding nodes in two parents are swapped each other by crossover rate of $P_{c}$ between the two parents (shadowed in Fig. 7). GNP evolves the fixed number of nodes, as I mentioned before, crossover is

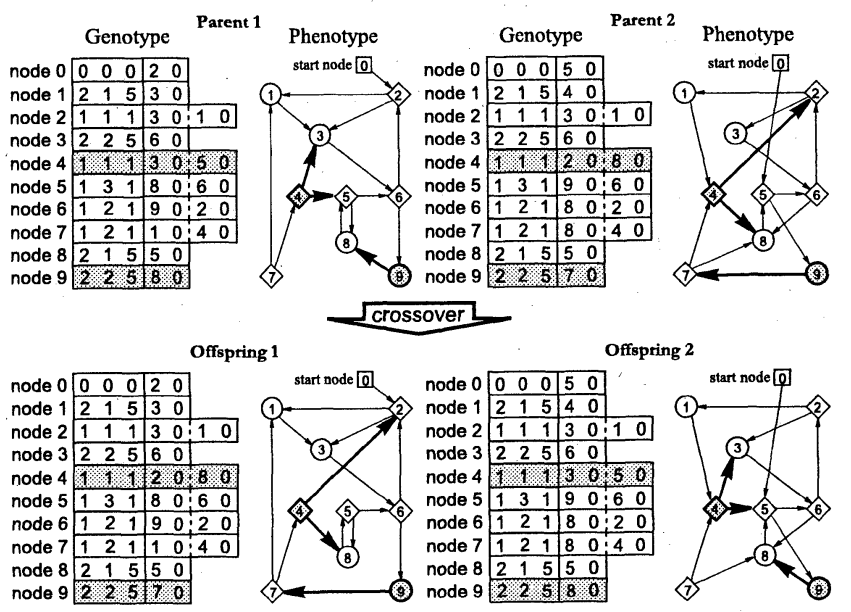

Fig. 7 Crossover in GNP
Fig. 6 Mutation in GNP

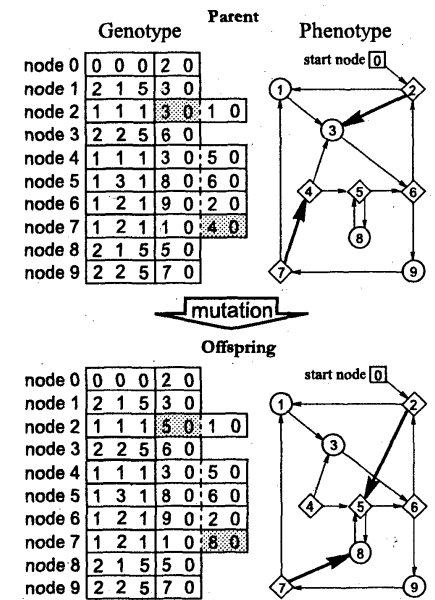


applied to the corresponding nodes selected uniformly in two parent genotypes as shown in Fig. 7 .

Note that these genetic operators will not change any node functions, they only change the connection among the nodes. Therefore GNP doesn't evolve the functions of the nodes, but evolves the connections between nodes.

\section{3 Comparison with other conventional methods}

GNP looks like Mealy type Finite State Automata, but nodes in GNP don't always denote the states. Furthermore, there are some functionally identical nodes in GNP. So, GNP is not a Finite State Automaton in a pure sense, instead it should be considered that GNP is an extension of EP for dealing with POMDP problems. When the problem is complex, the reactive policy will not work well in POMDPs. GNP works well even under such conditions, because the network based GNP does not produce the reactive policy, rather uses the past information implicitly.

The time required for judgement and processing $d_{i}$ and delay time $d_{i j}$ enable the realistic simulations of the real world. GNP is, therefore, the model of some kinds of general discrete systems.

GNP has a start node but no end node, therefore, the activated node is not forced to be transferred to the start node instead of the other methods, e.g., PADO. In addition tree structured GP executes from the root node at each step, so it may be fallen into the deadlocks because of the behaviors produced only by the current environments.

Furthermore, GP has a problem to be solved, that is, the bloat of the tree, especially the bloat problem becomes worse when using the multibranch tree. In the previous studies, the multibranch trees such as PROGN3 and IFLTH were used in GP, and splendid results were obtained ${ }^{2), 3)}$. But if almost all nodes have multibranches, it would severely cause the bloat.

The root node of the tree in GP contains many branches in lower levels, then usual genetic operators will be in trouble to evolve, unless the root node is selected appropriately at the beginning of generations. Some sophisticated GP techniques, ADF is one of these techniques, may overcome this problem, but very simple GP used in the simulations leads to the premature convergence as shown in the next section. On the contrary, the network with non-hierarchical structure doesn't have such problems even if the connection from the start node is considered. Actually, the changes of the connection from the start node are observed in the simulations in GNP, not only at the beginning of generations but also in the middle of generations.
In addition, the constraint of the depth of the tree often annoys GP researchers. The more the number of the kinds of JUDGEMENT NODE increases, the larger the depth of the tree should be in order to maintain the representation ability of the tree. The increase of depth causes the exponential enlargement of the search space, the occupation of large memory, and the increase of the calculation time. Many judgements in the tree take much execution time, which will be a severe problem in dynamic environments. On the other hand, it is expected that GNP can acquire the sophisticated structure by evolution in which only needed judgements are carried out according to necessity. Say when we walk around the city, do we always judge which direction to take to go to the shop? Judgement of which way to go just when occasion demands is enough. Of course, it is best to judge everything all the time if possible, but considering the judging time, the former will be the better strategy.

\section{Simulations}

In this section, an application using GNP is studied and compared with GP. Considering what stated before, the network based GNP can represent the behavior sequences of agents in POMDP, of course the POMDP cannot be solved by reactive policies. Moreover, it is highly expected to acquire the excellent behavior sequences for agents even when they are in complicated dynamical environments. The effects based on the structural difference between GNP and GP will be argued in this section.

\section{1 Simulation environments}

The tileworld (see section 3.1) is adopted to study the performance of GNP and GP.

The fitness value is defined as Eq.1 so as to consider 'how many tiles have been dropped?', 'how much have the tiles been approached to the holes?' and 'how fast have the task been completed?'. Dropped Tile means how many tiles have been dropped by agents within the time limit (in this paper, the time limit is 60 steps for each agent). Approached Distance means how much the tiles have been approached to the holes from the initial position. When an agent pushes a tile to a hole, Approached Distance is increased by 1 point. Rest Step is considered only when the agents completed the task. Rest Step is defined as the remaining time after completing the task. When agents get around 500 points, then the searching would be successful

$$
\begin{aligned}
\text { fitness } & =100 \times \text { Dropped Tile } \\
& +20 \times \text { Approached Distance }
\end{aligned}
$$




$$
+1 \times \text { Rest Step }
$$

\section{2 Conditions of GP}

Sometimes GP has been studied using numerical information as inputs for the terminal symbols and using arithmetic functions as the elementary functions ${ }^{10)}$. Nevertheless, GP in this paper uses if-then rules as elementary functions, and uses concrete actions as terminal symbols. For simplicity, the number of offspring by crossover and mutation is predefined. Furthermore, the best individual is preserved to the next generation as it is, that is, elite preservation strategy is adopted.

The population size is 301 . The number of offspring by crossover operator is 180 , the number of offspring by mutation operator is 120 and one individual is selected as an elite.

The terminal symbols are the set of $\{\mathrm{MF}, \mathrm{TR}, \mathrm{TL}, \mathrm{ST}\}$ and elementary functions are the set of $\{\mathrm{CF}, \mathrm{CR}, \mathrm{CL}$, CB, NTD, SNT, NHD, HTD $\}.\{C F, C R, C L, C B\}$ have five arguments which correspond to ffloor, tile, hole, barrier, agent\}, respectively. \{NTD, SNT, NHD, HTD $\}$ have four arguments and they correspond to \{right, front, left, back\}, respectively.

As described before, an example of the manually coded GP tree in the simulation is shown in Fig. 4. Note that such GP tree produces deterministic behavior rules, and considers current situation only.

The maximum depth of the tree is set to 5,6 or 7 , and they are appropriate values to compare GP with GNP. Then the total number of nodes of the tree in the simulation is around 300, 1000 and 3000 , respectively.

Tournament strategy is adopted as the selection method with its size being two, that is, select two individuals randomly then the superior one is selected as a parent for the next offspring. When mutation operator is carried out, one parent is selected by using tournament strategy once, then one offspring is created by mutation operator. When crossover operator is carried out, two parents is selected by using tournament strategy twice, then two offspring are created by crossover operator.

Only simple mutation operator and crossover operator are used to evolve individuals, no other sophisticated operators such as ADF are used. This may be the disadvantage for GP. But, we decided not to use such sophisticated operators to compare the fundamental differences between tree and graph.

\section{3 Conditions of GNP}

In the simulations of GNP, crossover and mutation are done in the same way of GP. The population size is 301 .
120 offspring are created by crossover operator, 180 offspring are created by mutation operator, and the best one individual is preserved to the next generation. The ratio of the offspring created by mutation and crossover differs from that in GP simulation. This is because GP generally makes much of crossover, on the contrary, GNP rather makes much of mutation.

Mutation operator affects all connections in GNP at probability $0.01\left(P_{m}=0.01\right)$, and uniform crossover is used $\left(P_{c}=0.4\right)$.

The size of the nodes is either 61 or 121 , the former denotes that one start node and twelve different kinds of nodes are used, each by fives [(MF, TR, TL, ST, CF, $\mathrm{CR}, \mathrm{CL}, \mathrm{CB}, \mathrm{NTD}, \mathrm{SNT}, \mathrm{NHD}, \mathrm{HTD}) \times 5+S]$, in the latter case one start node and twelve different kinds of nodes are prepared for their usage, each by tens $[(M F, T R, \cdots$, HTD) $\times 10+S$ ]. The former has 200 connections and the latter has 400 connections. Therefore, the comparisons with GP and GNP are done under appropriate conditions.

Other conditions are the same as GP. As described before, an example of manually coded GNP network in the simulation is shown in Fig. 3. Note that the network is interpreted successively (never return to the start node), this is the main difference from the tree structure. Then such GNP network does not produce simple behavior rules but behavior rules depending on the past record of its behaviors.

\subsection{Simulation results}

In this section, the comparison of GNP with GP is studied and discussed using the tileworld shown in Fig. 1.

\subsubsection{One tileworld simulations}

The comparison of GNP and GP in a relatively simple environment is done, that is, evolving the behavior sequences of agents is carried out using one tileworld (the left top tileworld in Fig. 1).

Fig. 8 shows the learning curves of GP with the maximum depth of the tree being 5,6 and 7, respectively. Each figure shows the maximum, mean and minimum fitness value over ten simulations, and also the following does. We can see that almost all runs could not acquire the desired behavior sequences. Contrary to our expectation, the more the maximum depth of the tree increases, the worse result seems to be acquired. And most of these simulations failed to evolve the desired tree.

Fig. 9 shows the results of GNP with the number of nodes being 61 and 121, respectively. As you can see, the simulation results show that GNP can evolve the networks successfully to adapt the tileworld. Furthermore, the convergence performance of GNP seems better than that of 


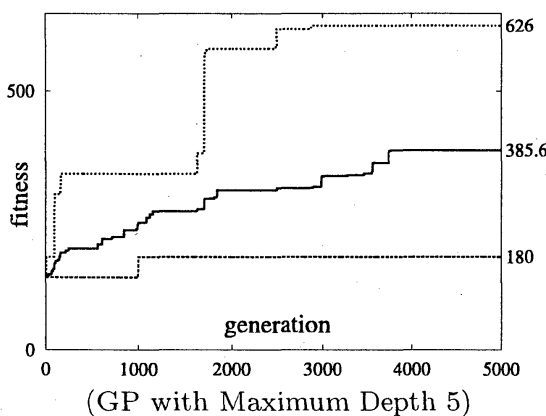

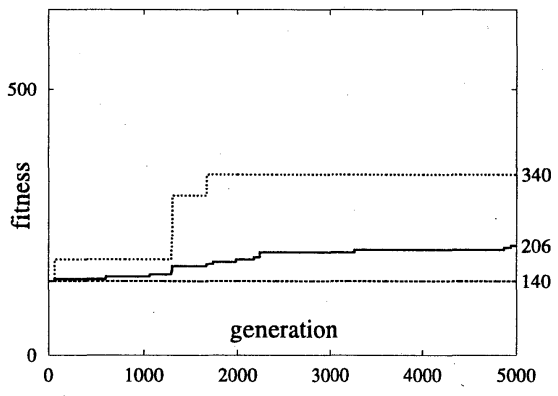

(GP with Maximum Depth 6)

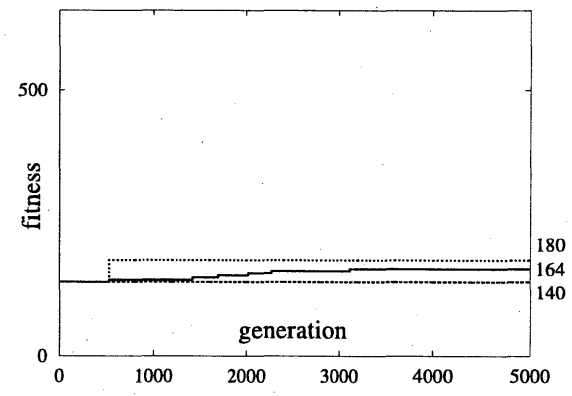

(GP with Maximum Depth 7)

Fig. 8 Learning curves of GP with maximum depth being 5, 6 and 7 using one tileworld
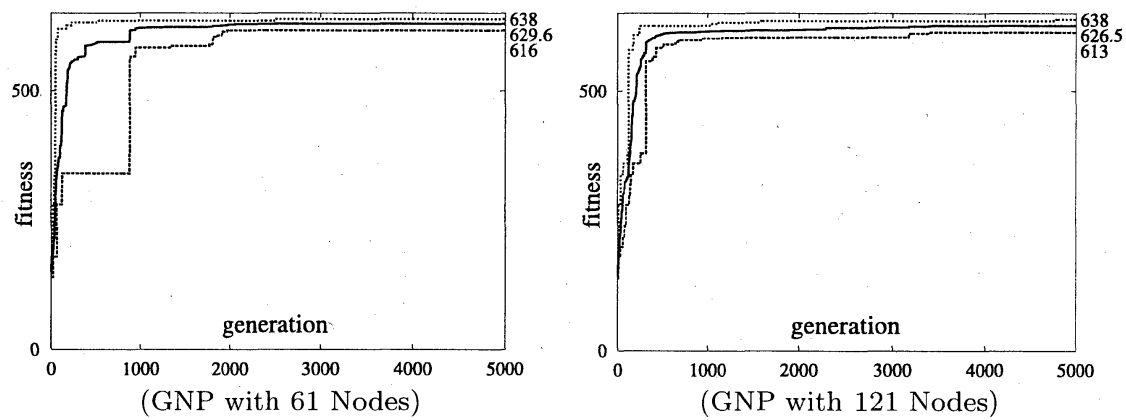

Fig. 9 Learning curves of GNP with 61 and 121 nodes using one tileworld

GP, especially the performance is more stable when the number of nodes is 121 .

\subsubsection{Ten tileworlds simulations}

In this simulation, the goal is to evolve the agents so as to adapt them to ten different tileworlds (see Fig. 1) simultaneously. The fitness value is the sum of the fitness values of each tileworld, then the searching would be successful when the fitness value is over around 5500 .

Fig. 10 shows the results of GP with maximum depth of the tree being 5, 6 and 7, respectively. And Fig. 11 shows the results of GNP with 61 nodes and 121 nodes.

The results of GP using ten tileworlds are better than those of using one tileworld. But sometimes GP stops evolving and falls into the premature convergence. In addition, the GP with 7-depth tree results in further worse evolution. On the contrary, GNP can often gain very high fitness by evolving individuals, and relatively high-average fitness values are acquired.

An example of agents' behaviors performed by GP and GNP are shown in Fig. 12 and Fig. 13, respectively. These figures show the satisfied results of GP and GNP. In Fig. 12 (GP), we can see that judgements in each step are very similar to each other, and the agents always judge NTD firstly. The agents perform four judgements before taking an action in almost all steps. On the contrary, various kinds of judgements are performed in each step in
Fig. 13 (GNP). We can see that the GNP agents generally perform fewer judgements than the GP agents do in each step. This is because the GNP agents can utilize the past information stored in the network, while the GP agents can not. The GP agents can only behave according to the current situation, then they tend to fall into the deadlock. Actually, the GP agent 0 hardly moves at the beginning (from step 0 to step 10), and the GP agent 2 clearly falls into the deadlock from step 10 to step 20. They can not escape from the deadlock until other agents change the situation.

\subsubsection{Simulations using enhanced JUDGEMENT NODEs}

In this simulation, the functions of some JUDGEMENT NODEs are changed from the previous simulations. As described in section 3 , it is difficult to use GP when the number of the branches of the nodes is large, because the total number of nodes required becomes exponentially large. On the other hand, it is fairly easy to increase the number of branches of the nodes in GNP because not the total number of nodes but only the total number of connections increases linearly.

Considering the above, the following four JUDGEMENT NODEs such as NTD, SNT, NHD and HTD originally having 4 connections \{right, front, left, back \} are changed to have 8 connections \{right, front, left, back, precisely right, precisely front, precisely left, precisely back $\}$. In other words, 

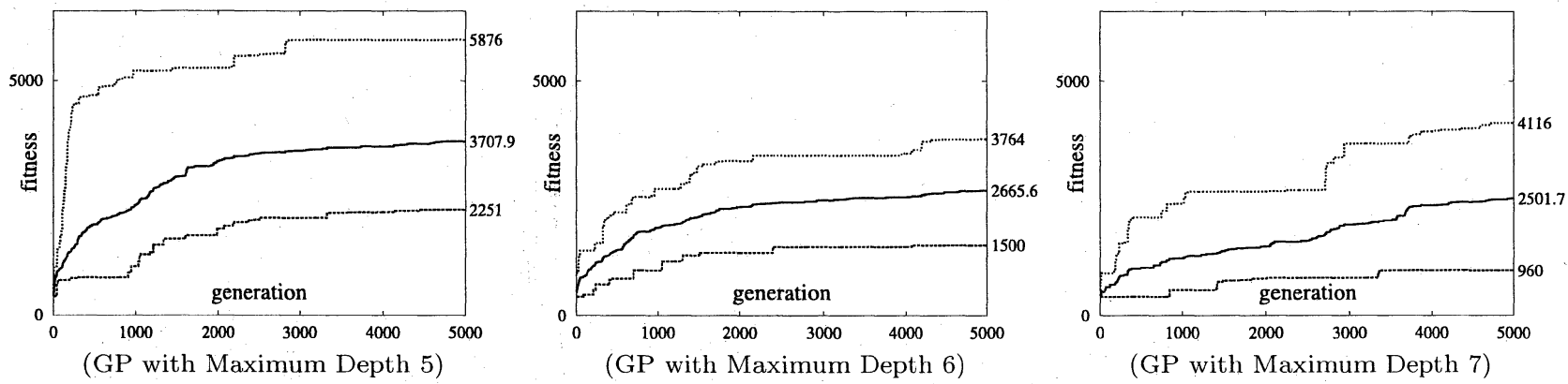

(GP with Maximum Depth 7)

Fig. 10 Learning curves of GP with maximum depth being 5, 6 and 7 using ten tileworlds
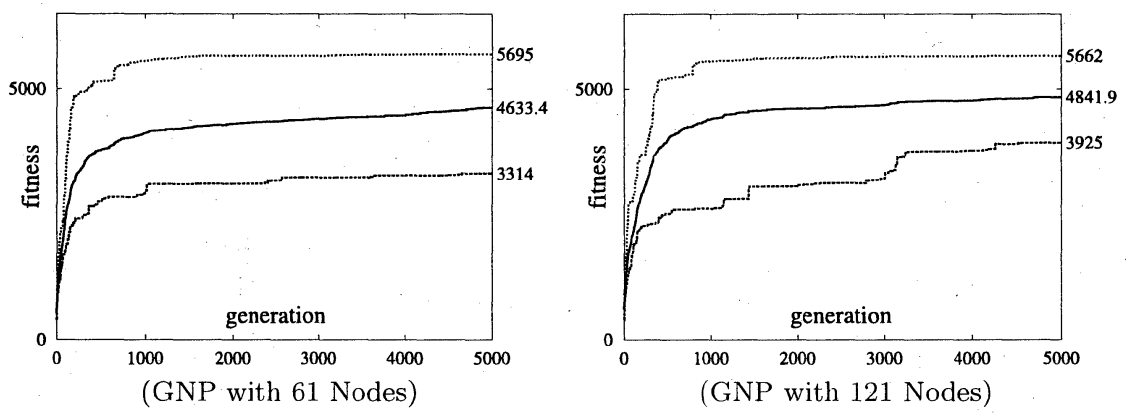

Fig. 11 Learning curves of GNP with 61 and 121 nodes using ten tileworlds

the capability of sensing direction of agents is enhanced in this simulation.

The simulation results are shown in Fig. 14, where the better individuals tend to be acquired by such enhanced JUDGEMENT NODEs. In this way, it is clarified that GNP can evolve the individuals successfully, even when the functions with many branches are used.

\section{Conclusions}

In this paper, a new evolutionary method named GNP (Genetic Network Programming) is proposed and GNP is compared with GP using the typical tileworld benchmark problem. The distinguished point of GNP is a network based structure instead of a tree structure in GP.

For this particular problem of the tileworld and the parameters tested, it has been clarified that GNP can evolve more effectively than GP, as a result, premature convergence hardly occurs in GNP compared with GP. It is also shown that the network based GNP can evolve even in the POMDP problems.

\section{References}

1) J.H. Holland: Adaptation in Natural and Artificial Systems, University of Michigan Press (1975), MIT Press (1992)

2) J.R. Koza: Genetic Programming: On the Programming of Computers by Means of Natural Selection, MIT Press (1992)
3) J.R. Koza: Genetic Programming II: Automatic Discovery of Reusable Subprograms, MIT Press (1994)

4) L.J. Fogel, A.J. Owens and M.J. Walsh: Artificial Intelligence through Simulated Evolution, Wiley (1966)

5) D.B. Fogel: An introduction to simulated evolutionary optimization, IEEE Trans. on Neural Networks, 5-1, 3/14 (1994)

6) A. Teller and M. Veloso: PADO: Learning Tree-structured Algorithms for Orchestration into an Object Recognition System, Carnegie Mellon University Technical Report Library (1995)

7) J. Miller and P. Thomson: Cartesian Genetic Programming, Genetic Programming, Proceedings of EuroGP' (2000)

8) M.E. Pollack and M. Ringuette: Introducing the tileworld: Experimentally evaluating agent architectures. In Proceedings of the Conference of the American Association for Artificial Intelligence, 183/189 (1990)

9) C.V. Goldman and J.S. Rosenschein: Emergent coordination through the use of cooperative state-changing rules. In Proceedings of the Twelfth National Conference on Artificial Intelligence, 408/413, Seattle, WA. (1994)

10) H. Iba: Evolutionary Learning of Communicating Agents, Information Science, 108-1-4, 181/205, Elsevier (1998)

11) H. Katagiri, K. Hirasawa and J. Hu: Genetic Network Programming -Application to Intelligent Agents-, Proc. of IEEE International Conference on System, Man and Cybernetics, 3829/3834 (2000)

12) K. Hirasawa, M. Okubo, J. Hu and J. Murata: Comparison between Genetic Network Programming (GNP) and Genetic Programming (GP), Proc. of CEC International Conference, 2, 1276/1282 (2001)

13) H. Katagiri, K. Hirasawa, J. Hu and J. Murata: Network Structure Oriented Evolutionary Model - Genetic Net- 


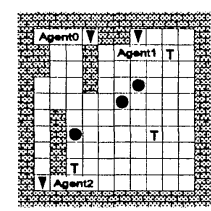

step0

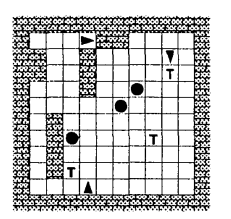

step5

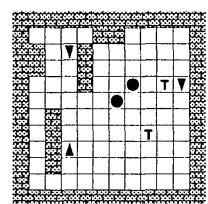

step10

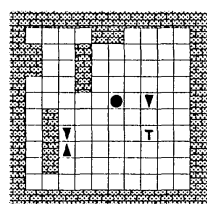

step15

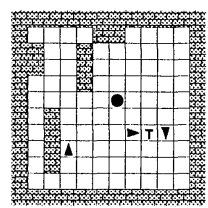

step20

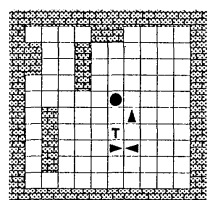

step 15

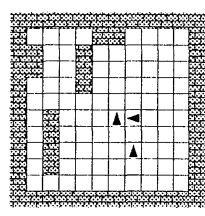

step28

[Agent 0 (left top)]

$\mathrm{NHD}, \mathrm{CF}, \mathrm{CL}, \mathrm{SNT}, \mathrm{TR} \rightarrow \mathrm{NHD}, \mathrm{CF}, \mathrm{NTD}, \mathrm{CL}, \mathrm{MF} \rightarrow \mathrm{NHD}, \mathrm{CF}, \mathrm{NTD}, \mathrm{NHD}, \mathrm{TL} \rightarrow \mathrm{NHD}, \mathrm{NTD}, \mathrm{NTD}, \mathrm{CL}, \mathrm{TL} \rightarrow \mathrm{NHD}, \mathrm{CF}, \mathrm{NTD}, \mathrm{HTD}, \mathrm{MF} \quad \rightarrow$ $\mathrm{NHD}, \mathrm{NTD}, \mathrm{CF}, \mathrm{CR}, \mathrm{TL} \rightarrow \mathrm{NHD}, \mathrm{CF}, \mathrm{NTD}, \mathrm{CR}, \mathrm{TL} \rightarrow \mathrm{NHD}, \mathrm{CF}, \mathrm{NTD}, \mathrm{CL}, \mathrm{MF} \rightarrow \mathrm{NHD}, \mathrm{CF}, \mathrm{NTD}, \mathrm{SNT}, \mathrm{TL} \rightarrow \mathrm{NHD}, \mathrm{NTD}, \mathrm{CF}, \mathrm{CF}, \mathrm{MF} \rightarrow$ $\mathrm{NHD}, \mathrm{CF}, \mathrm{NTD}, \mathrm{SNT}, \mathrm{MF} \rightarrow \mathrm{NHD}, \mathrm{CF}, \mathrm{NTD}, \mathrm{SNT}, \mathrm{MF} \rightarrow \mathrm{NHD}, \mathrm{CF}, \mathrm{NTD}, \mathrm{SNT}, \mathrm{MF} \rightarrow \mathrm{NHD}, \mathrm{CF}, \mathrm{NTD}, \mathrm{SNT}, \mathrm{MF} \rightarrow \mathrm{NHD}, \mathrm{CF}, \mathrm{NTD}, \mathrm{SNT}, \mathrm{MF} \quad \rightarrow$ $\mathrm{NHD}, \mathrm{CF}, \mathrm{CL}, \mathrm{CL}, \mathrm{TL} \rightarrow \mathrm{NHD}, \mathrm{NTD}, \mathrm{CF}, \mathrm{CF}, \mathrm{MF} \rightarrow \mathrm{NHD}, \mathrm{CF}, \mathrm{NTD}, \mathrm{CL}, \mathrm{MF} \rightarrow \mathrm{NHD}, \mathrm{CF}, \mathrm{NTD}, \mathrm{CL}, \mathrm{MF} \rightarrow \mathrm{NHD}, \mathrm{CF}, \mathrm{NTD}, \mathrm{CL}, \mathrm{MF} \rightarrow$ $\mathrm{NHD}, \mathrm{CF}, \mathrm{CL}, \mathrm{SNT}, \mathrm{TR} \rightarrow \mathrm{NHD}, \mathrm{CF}, \mathrm{NTD}, \mathrm{CF}, \mathrm{MF} \rightarrow \mathrm{NHD}, \mathrm{CF}, \mathrm{NTD}, \mathrm{CL}, \mathrm{TL} \rightarrow \mathrm{NHD}, \mathrm{CF}, \mathrm{NTD}, \mathrm{NHD}, \mathrm{TL} \rightarrow \mathrm{NHD}, \mathrm{NTD}, \mathrm{NTD}, \mathrm{CL}, \mathrm{TL} \rightarrow$ $\mathrm{NHD}, \mathrm{CF}, \mathrm{CL}, \mathrm{HTD}, \mathrm{TR} \rightarrow \mathrm{NHD}, \mathrm{NTD}, \mathrm{NTD}, \mathrm{CL}, \mathrm{TL} \rightarrow \mathrm{NHD}, \mathrm{CF}, \mathrm{NTD}, \mathrm{CR}, \mathrm{TR}$

[Agent 1 (right top)]

$\mathrm{NHD}, \mathrm{NTD}, \mathrm{NTD}, \mathrm{CL}, \mathrm{TL} \rightarrow \mathrm{NHD}, \mathrm{CF}, \mathrm{NTD}, \mathrm{HTD}, \mathrm{MF} \quad \rightarrow \quad \mathrm{NHD}, \mathrm{CF}, \mathrm{NTD}, \mathrm{HTD}, \mathrm{MF} \quad \rightarrow \quad \mathrm{NHD}, \mathrm{CF}, \mathrm{NTD}, \mathrm{CR}, \mathrm{TR} \quad \rightarrow \quad \mathrm{NHD}, \mathrm{NTD}, \mathrm{CF}, \mathrm{MF} \quad \rightarrow$ $\mathrm{NHD}, \mathrm{NTD}, \mathrm{CF}, \mathrm{MF} \rightarrow \mathrm{NHD}, \mathrm{CF}, \mathrm{NTD}, \mathrm{CL}, \mathrm{TL} \quad \rightarrow \quad \mathrm{NHD}, \mathrm{CF}, \mathrm{NTD}, \mathrm{CR}, \mathrm{MF} \quad \rightarrow \quad \mathrm{NHD}, \mathrm{CF}, \mathrm{NTD}, \mathrm{CL}, \mathrm{TR} \quad \rightarrow \quad \mathrm{NHD}, \mathrm{CF}, \mathrm{NTD}, \mathrm{HTD}, \mathrm{MF} \quad \rightarrow$ $\mathrm{NHD}, \mathrm{CF}, \mathrm{NTD}, \mathrm{CR}, \mathrm{TR} \rightarrow \mathrm{NHD}, \mathrm{NTD}, \mathrm{CF}, \mathrm{MF} \rightarrow \mathrm{NHD}, \mathrm{NTD}, \mathrm{CF}, \mathrm{MF} \quad \rightarrow \quad \mathrm{NHD}, \mathrm{NTD}, \mathrm{NTD}, \mathrm{CL}, \mathrm{TL} \quad \rightarrow \quad \mathrm{NHD}, \mathrm{CF}, \mathrm{NTD}, \mathrm{HTD}, \mathrm{MF} \quad \rightarrow$ $\mathrm{NHD}, \mathrm{CF}, \mathrm{NTD}, \mathrm{HTD}, \mathrm{MF} \rightarrow \mathrm{NHD}, \mathrm{CF}, \mathrm{NTD}, \mathrm{CL}, \mathrm{TL} \quad \rightarrow \quad \mathrm{NHD}, \mathrm{CF}, \mathrm{NTD}, \mathrm{CR}, \mathrm{MF} \quad \rightarrow \quad \mathrm{NHD}, \mathrm{CF}, \mathrm{NTD}, \mathrm{CR}, \mathrm{TR} \quad \rightarrow \quad \mathrm{NHD}, \mathrm{CF}, \mathrm{NTD}, \mathrm{HTD}, \mathrm{MF} \quad \rightarrow$ $\mathrm{NHD}, \mathrm{CF}, \mathrm{NTD}, \mathrm{CR}, \mathrm{TR} \rightarrow \mathrm{NHD}, \mathrm{NTD}, \mathrm{CF}, \mathrm{MF} \rightarrow \mathrm{NHD}, \mathrm{NTD}, \mathrm{CF}, \mathrm{MF} \quad \rightarrow$ NHD,CF,NTD,CL,TR $\rightarrow \quad$ NHD,NTD,NTD,CL,MF $\rightarrow$ NHD,CF,NTD,NHD,TL $\rightarrow$ NHD,NTD,NTD,CL,TL $\rightarrow$ NHD,CF,NTD,CR,TR

[Agent 2 (left bottom)]

$\mathrm{NHD}, \mathrm{CF}, \mathrm{NTD}, \mathrm{SNT}, \mathrm{TL} \quad \rightarrow$ NHD,CF,NTD,CL,MF $\rightarrow$ NHD,CF,NTD,SNT,MF $\rightarrow$ NHD,CF,NTD,SNT,MF $\rightarrow \quad$ NHD,CF,NTD,NHD,TL $\rightarrow$ $\mathrm{NHD}, \mathrm{NTD}, \mathrm{NTD}, \mathrm{CL}, \mathrm{TL} \rightarrow \mathrm{NHD}, \mathrm{CF}, \mathrm{NTD}, \mathrm{HTD}, \mathrm{MF} \rightarrow \mathrm{NHD}, \mathrm{CF}, \mathrm{NTD}, \mathrm{CR}, \mathrm{TR} \rightarrow \mathrm{NHD}, \mathrm{NTD}, \mathrm{CF}, \mathrm{MF} \quad \rightarrow \quad \mathrm{NHD}, \mathrm{NTD}, \mathrm{CF}, \mathrm{MF} \quad \rightarrow$ NHD,NTD,HTD,CL,ST $\rightarrow$ NHD,NTD,HTD,CL,ST $\rightarrow$ NHD,NTD,HTD,CL,ST $\rightarrow$ NHD,NTD,HTD,CL,ST $\rightarrow \quad$ NHD,NTD,HTD,CL,ST $\rightarrow$ NHD,NTD,HTD,CL,ST $\rightarrow$ NHD,NTD,HTD,CL,ST $\rightarrow$ NHD,NTD,HTD,CL,ST $\rightarrow$ NHD,NTD,HTD,CL,ST $\rightarrow \quad$ NHD,NTD,HTD,CL,ST $\rightarrow$ $\mathrm{NHD}, \mathrm{NTD}, \mathrm{HTD}, \mathrm{CL}, \mathrm{ST} \rightarrow \mathrm{NHD}, \mathrm{NTD}, \mathrm{HTD}, \mathrm{CB}, \mathrm{TR} \quad \rightarrow \quad \mathrm{NHD}, \mathrm{CF}, \mathrm{NTD}, \mathrm{CL}, \mathrm{MF} \quad \rightarrow \quad \mathrm{NHD}, \mathrm{CF}, \mathrm{NTD}, \mathrm{CL}, \mathrm{MF} \quad \rightarrow \quad \mathrm{NHD}, \mathrm{CF}, \mathrm{NTD}, \mathrm{SNT}, \mathrm{MF} \quad \rightarrow$ $\mathrm{NHD}, \mathrm{CF}, \mathrm{CL}, \mathrm{NHD}, \mathrm{TL} \rightarrow \mathrm{NHD}, \mathrm{NTD}, \mathrm{CF}, \mathrm{MF} \rightarrow \mathrm{NHD}, \mathrm{NTD}, \mathrm{CF}, \mathrm{MF}$

Fig. 12 An example of agents' behaviors performed by GP

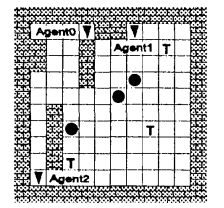

step0

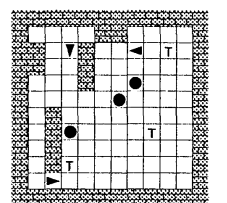

step5

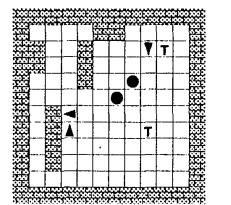

step10

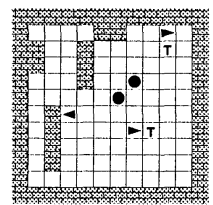

step15

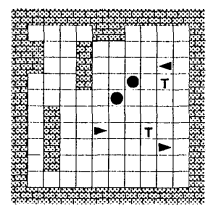

step20

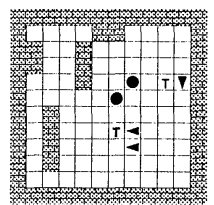

step 15

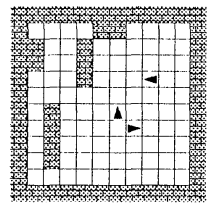

step28

[Agent 0 (left top)] NTD,NTD,NHD,NHD, CB

$\mathrm{CB}, \mathrm{CF}, \mathrm{MF}$

$\rightarrow \mathrm{TR}$

$\rightarrow \quad \mathrm{NTD}, \mathrm{CF}, \mathrm{MF}$

$\rightarrow \quad \mathrm{NTD}, \mathrm{CF}, \mathrm{MF}$

$\rightarrow$ NTD,CF,MF

$\mathrm{CF}, \mathrm{SNT}, \mathrm{NHD}, \mathrm{CR}, \mathrm{TR}$

$\rightarrow \mathrm{NTD}, \mathrm{CL}, \mathrm{HTD}, \mathrm{CL}, \mathrm{CR} \rightarrow \mathrm{ST}$

$\rightarrow \quad \mathrm{NTD}, \mathrm{CB}, \mathrm{TL}$

$\rightarrow \mathrm{NTD}, \mathrm{CF}, \mathrm{MF}$

$\rightarrow$ SNT,TL

$\mathrm{CF}, \mathrm{CB}, \mathrm{TL}$

NTD, CF, MF

$\rightarrow \quad \mathrm{CR}, \mathrm{MF}$

$\rightarrow$ NTD,CF,MF

$\rightarrow$ NHD,NHD,CB,TL

$\rightarrow \quad \mathrm{CR}, \mathrm{CB}, \mathrm{NHD}, \mathrm{MF}$

$\rightarrow \mathrm{NTD}, \mathrm{CF}, \mathrm{NHD}, \mathrm{CF}, \mathrm{TR} \rightarrow \mathrm{CR}, \mathrm{CB}, \mathrm{CF}, \mathrm{MF}$

$\mathrm{CB}, \mathrm{CF}, \mathrm{MF}$

$\rightarrow$ NTD,TR

[Agent 1 (right top)]

NTD,NTD,NHD,NTD, TR $\rightarrow$ CF,SNT,MF

$\mathrm{CF}, \mathrm{CF}, \mathrm{HTD}, \mathrm{CL}, \mathrm{TR} \rightarrow \mathrm{SNT}, \mathrm{TR}$

TR

NTD, TR

$\rightarrow \mathrm{CR}, \mathrm{CB}, \mathrm{TR}$

$\rightarrow \quad \mathrm{CR}, \mathrm{CB}, \mathrm{NHD}, \mathrm{MF}$

TR

$\rightarrow \mathrm{CR}, \mathrm{CB}, \mathrm{TR}$

NTD,TR

$\rightarrow \quad \mathrm{CR}, \mathrm{CB}, \mathrm{NHD}, \mathrm{MF}$

$\rightarrow \mathrm{CR}, \mathrm{CB}, \mathrm{CF}, \mathrm{NHD}, \mathrm{MF}$

$\rightarrow$ NTD,CF,NHD,MF

$\rightarrow$ NHD,CB,TL

$\rightarrow \mathrm{CR}, \mathrm{MF}$

$\rightarrow \mathrm{CB}, \mathrm{CF}, \mathrm{MF}$

$\rightarrow \mathrm{NTD}, \mathrm{CF}, \mathrm{NHD}, \mathrm{MF}$

$\rightarrow \mathrm{CB}, \mathrm{CF}, \mathrm{MF}$

$\rightarrow$ NTD,CF,NHD,MF

[Agent 2 (left bottom)]

\section{NTD,NTD,NHD, TL}

$\mathrm{CR}, \mathrm{MF}$

$\rightarrow \mathrm{CB}, \mathrm{MF}$

$\rightarrow$ NHD,NHD,CB,TL

$\rightarrow \quad \mathrm{CR}, \mathrm{MF}$

$\rightarrow$ NTD,CF,NHD,TR

$\rightarrow$ NTD,TR

$\rightarrow \quad \mathrm{NTD}, \mathrm{CF}, \mathrm{NHD}, \mathrm{TR}$

$\rightarrow$ NTD,TR

\section{$\rightarrow \quad$ HTD,SNT,MF}

$\rightarrow$ NTD,TR

$\rightarrow \quad \mathrm{CR}, \mathrm{NTD}, \mathrm{ST}$

$\rightarrow \quad \mathrm{NTD}, \mathrm{CF}, \mathrm{MF}$

$\rightarrow$ NTD,CB,CB,TR

$\rightarrow \quad \mathrm{NHD}, \mathrm{NTD}, \mathrm{TR}$

$\rightarrow \mathrm{CF}, \mathrm{CF}, \mathrm{HTD}, \mathrm{CF}, \mathrm{HTD} \rightarrow$

$\rightarrow \mathrm{CR}, \mathrm{CB}, \mathrm{CF}, \mathrm{MF}$

$\rightarrow \quad \mathrm{CF}, \mathrm{CF}, \mathrm{HTD}, \mathrm{CF}, \rightarrow$

$\rightarrow \mathrm{CR}, \mathrm{CB}, \mathrm{CF}, \mathrm{MF}$

$\rightarrow \quad \mathrm{NHD}, \mathrm{NHD}, \mathrm{CB}, \mathrm{TL}$

$\rightarrow \quad \mathrm{CR}, \mathrm{CB}, \mathrm{NHD}, \mathrm{MF}$

$\rightarrow$ NTD, CF,NHD,MF

$\rightarrow \mathrm{NTD}, \mathrm{CF}, \mathrm{MF}$

$\rightarrow$ HTD,MF

$\rightarrow \mathrm{CR}, \mathrm{CB}, \mathrm{CF}, \mathrm{NHD}, \mathrm{MF}$

$\rightarrow \quad \mathrm{NTD}, \mathrm{CF}, \mathrm{CF}, \mathrm{TR}$

$\rightarrow$ NTD,NHD,MF

$\rightarrow$ NTD,CF,MF

$\rightarrow \quad \mathrm{CB}, \mathrm{CF}, \mathrm{MF}$

$\rightarrow \quad \mathrm{NHD}, \mathrm{CB}, \mathrm{TL}$

$\rightarrow \mathrm{TR}$

Fig. 13 An example of agents' behaviors performed by GNP 

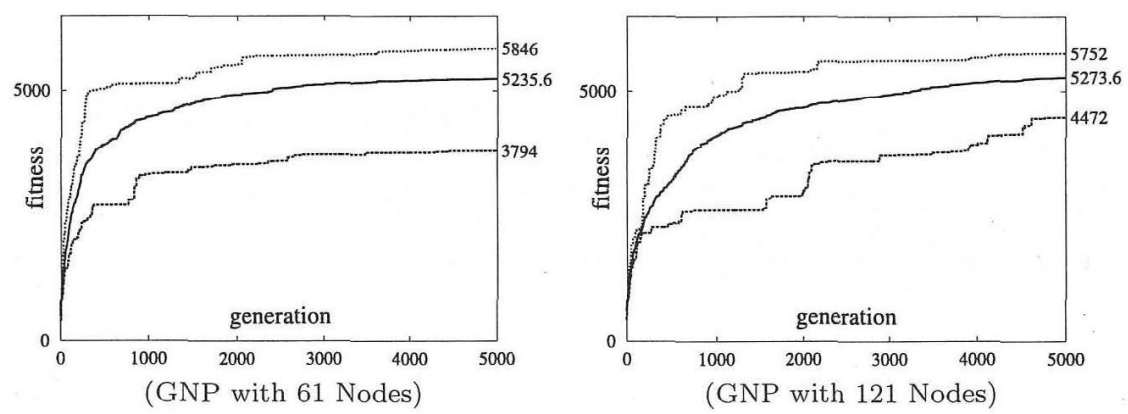

Fig. 14 GNP with enhanced functions with 61 and 121 nodes using ten tileworlds

work Programming - and Its Comparison with Genetic Programming, Proc. of GECCO International Conference (2001)

14) K. Hirasawa, M. Okubo, H. Katagiri, J. Hu and J. Murata: Comparison between Genetic Network Programming using evolution of ant's behaviors, Transactions of the Institute of Electrical Engineers of Japan, 121-C-6, 1001/1009 (2001) (in Japanese)

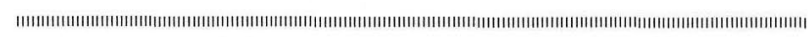
Hironobu Katagiri (Student Member)

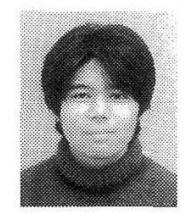

He received the M.S. degree in Electrical and Electronic Systems Engineering from Kyushu University in 2000. His current research interests are evolutionary computation, planning and their applications. He is a member of the Institute of Electrical Engineers of Japan.

\section{Kotaro Hirasawa (Member)}

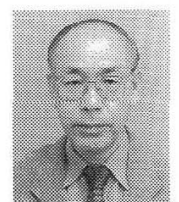

He received the M.S. degree in Electrical Engineering from Kyushu University in 1966. From April 1966, he served in Hitachi Lab. of Hitachi Ltd., and in 1989 he was a vice president of IItachi Lab.. From August 1991 to November 1992, he served in Omika Factory of Hitachi Ltd.. Since December 1992, he has been a professor in the faculty of Engineering, Kyushu University. Now he belongs to the Graduate School of Information Science and Electrical Engineering, Kyushu University. He is a member of the Institute of Electrical Engineers of Japan, a member of IEEE.

\section{Jinglu Hu (Member)}

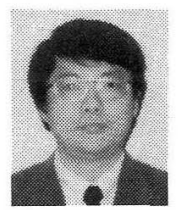

He received the M.Sci. degree in 1986 from Zhongshan University, China and the Ph.D degree in 1997 from Kyushu Institute of Technology, Japan. From 1988 to 1993, he was a Lecturer in Zhongshan University. Since 1997, he has been a Research Associate at Kyushu University. His current research interests are learning network theory, system identification and their applications. Dr. Hu is a member of the Institute of Electrical Engineers of Japan.

\section{Junichi Murata (Member)}

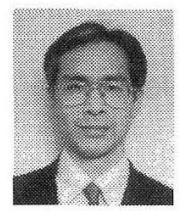

He received the D.Eng. degree in Electrical Engineering from Kyushu University in 1986. From 1986 to March 1988, he was a Research Associate at Kyushu University. From April 1988, he is an Associate Professor at Kyushu University. Now he belongs to the Graduate School of Information Science and Electrical Engineering, Kyushu University. He is a member of the Institute of Electrical Engineers of Japan, a member of ISCIE and a member of IEEE.

\section{Michitaka Kosaka (Member)}

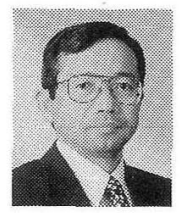

He received the M.S. degree in 1977, the $\mathrm{Ph} . \mathrm{D}$ degree in 1984 respectively from Kyoto University. He joined Systems Development Laboratory, Hitachi Ltd. in 1977. Since then, he has been doing research and development on Large-scale System Engineering. Now he is a general manager of System Development Laboratory.

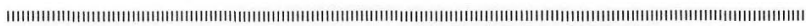

